\title{
Negative linear compressibility in common materials
}

\author{
W. Miller, ${ }^{1}$ K.E. Evans ${ }^{1}$, A. Marmier ${ }^{1, a)}$ \\ ${ }^{1}$ College of Engineering Mathematics and Physical Science, University of Exeter, Exeter, EX4 \\ $4 Q F, U K$
}

Negative linear compressibility (NLC) is still considered an exotic property, only observed in a few obscure crystals. The vast majority of materials compress axially in all directions when loaded in hydrostatic compression. However a few materials have been observed which expand in one or two directions under hydrostatic compression. At present, the list of materials demonstrating this unusual behaviour is confined to a small number of relatively rare crystal phases, biological materials and designed structures, and the lack of widespread availability hinders promising technological applications. Using improved representations of elastic properties, this study revisits existing databases of elastic constants and identifies several crystals missed by previous reviews. More importantly, several common materials -drawn polymers, certain types of paper and wood, and carbon fibre laminates- are found to display NLC. We show that NLC in these materials originates from the misalignment of polymers/fibres. Using a beam model we propose that maximum NLC is obtained for misalignment of $26^{\circ}$. The existence of such widely available materials increases significantly the prospects for applications of NLC.

Keywords: Negative Linear Compressibility, Elastic Properties, Mechanical Properties, Analytical Methods, Composites. 
a) Author to whom correspondence should be addressed. Electronic mail: A.S.H.Marmier@exeter.ac.uk 
First identified in tellurium in $1922^{1}$, negative linear compressibility (NLC) has long been considered to be confined to rare crystal phases and been the subject of little scientific or industrial interest. The recent discovery of materials and structures ${ }^{2-8}$ displaying NLC suggest that this property is not as rare as previously considered and may lead to interesting developments. The main aim of this study is to re-examine previously published elastic properties and to identify NLC in common materials.

Materials that display unusual properties are currently the focus of a great deal of research interest: negative Poisson's ratio ${ }^{9}$ structures for honeycomb sandwich cores and composites, negative thermal expansion ${ }^{10}$ for satellite structures and microchip packaging, negative refractive index for optics ${ }^{11}$ and negative stiffness ${ }^{12}$ for earthquake proof buildings.

Compressibility describes the behaviour of a material or structure under pressure, positive (compressive) or negative (tensile). Volumetric compressibility defines the volume change in a material under applied hydrostatic pressure, whilst linear compressibility describes a change along a specified axis of a material. Negative linear compressibility occurs when a material behaves contrary to expectation by conventional elasticity theory under the application of hydrostatic pressure e.g. lengthening under hydrostatic compression, as shown in Figure 1. As this unusual property may be accompanied by a corresponding large positive linear compressibility along another axis, this may lead to the situation where a material with entirely conventional volume compressibility has both a large positive and negative or zero compressibilities that are not captured by considering only the volume compressibility.

The current range of NLC materials is limited - the early review by Baughman $e a^{2}{ }^{2}$ listed 13 materials and the recent review by Cairns et al. ${ }^{7}$ mentions 30 materials, with only two being duplicated with ${ }^{2}-$. NLC has been observed in crystalline phases ${ }^{2,4,7,8,13}$, biological materials ${ }^{2,14}$, designed structures ${ }^{3,15}$ and systems of confined materials/structures ${ }^{16}$. To date, there has been little commercial interest in this property, which is at least partially due to the absence of commonly available 'engineering' materials. In this context, we consider materials to be common if they can be produced in large quantity and do not have to be in single-crystal form to show NLC. Such materials are conspicuous by their absence from lists of NLC materials: even the most recent review ${ }^{7}$ does not mention any.

The letter is organised as follow: after defining NLC, we identify several materials that exhibit NLC, including common materials such as paper and drawn polymers. The mechanism of 
NLC in these can be explained by a simple mechanistic model based on beam theory. Finally, we discuss several realistic applications where the property may provide increased performance over conventional materials.

The volume compressibility $\beta_{V}$ is defined from the relative change in volume $V$ due to isotropic pressure $p$ as $-\frac{1}{V} \frac{d V}{d p}$, the temperature remaining constant. It is an invariant of the compliance tensor $S$, and can be written using Voigt's notation as

$$
\beta_{V}=\left(S_{11}+S_{22}+S_{33}\right)+2\left(S_{12}+S_{23}+S_{31}\right) \text {. }
$$

For isotropic materials, it is linked to Young's modulus $E$ and Poisson's ratio $v$ by $\beta_{V}=$ $\frac{3(1-2 v)}{E}$. Its value varies from a few $\mathrm{TPa}^{-1}$ for a hard material such as diamond $\left(2.1 \mathrm{TPa}^{-1}\right)$ to a few hundreds for soft polymers such as polystyrene $\left(330 \mathrm{TPa}^{-1}\right)$, up to a thousand or above for very compliant materials such as foams.

Similarly, the linear compressibility at constant temperature $\beta_{l}$ can be defined by $-\frac{1}{l} \frac{d l}{d p}$ or $-\frac{\varepsilon}{\Delta p}$ where $p$ is the applied hydrostatic pressure, $l$ and $d l$ the part's dimension and elementary displacement in the direction of interest, and $\varepsilon$ the strain. For isotropic materials, $\beta_{l}=\beta_{V} / 3$.

For orthorhombic/orthotropic structures, the linear compressibility for a given direction (unit vector $\boldsymbol{n}$ ) can be expressed as ${ }^{17}$

$$
\beta_{l}=\left(S_{11}+S_{12}+S_{13}\right) n_{1}^{2}+\left(S_{21}+S_{22}+S_{23}\right) n_{2}^{2}+\left(S_{31}+S_{32}+S_{33}\right) n_{3}^{2}
$$

where the $S_{i j}$ are non-zero components from the compliance tensor written using Voigt's notation and the $n_{i}$ are the components of the unit vector $\boldsymbol{n}$. In direction 1 , this reduces to $\beta_{l 1}=\left(1-v_{21}-v_{31}\right) / E_{1}$, which indicates that NLC is associated with large positive Poisson's ratio.

At this point, it is useful to comment on the relation between NLC and the related property of stretch densification highlighted by earlier studies ${ }^{2}$. For a start, it would be more accurate to compare negative linear expansivity and stretch densification. Many materials have the same elastic properties in tension and compression, but this is by no means generic, as exemplified by fibres and their composites. With this caveat in mind, it is sometimes easier to visualise stretch densification to elucidate mechanisms and discover candidate materials and applications.

Two main procedures can be used to identify NLC materials. The first one consists in calculating the linear compressibility in any direction from a full set of elastic constants obtained 
from experiments or calculations. We use the ElAM code ${ }^{18}$ which automates the derivation of tensor based elastic properties from databases of elastic stiffness or compliances; two graphical examples are shown in Figure $\mathrm{S} 1^{19}$. The second approach is more direct and consists in measuring the dimension change under pressure with X-ray or neutron diffraction. Usually, only lattice dimensions are measured, and off-axis LC which may be larger might be missed. The first method, from now referred to as elastic, is limited to the elastic limit and to small strain. The direct method reaches large strain, up to $5 \%$ for $\operatorname{Ag}_{3}\left[\mathrm{Co}(\mathrm{CN})_{6}\right]-\mathrm{II}^{4}$. It also allows the identification of NLC at high pressure, occasionally for materials with conventional LC at standard pressure. Where the two methods have been applied $\left(\mathrm{LaNbO}_{4}\right.$ and $\left.\mathrm{Se}\right)$, the elastic and direct values for NLC are comparable at $-23.3 \mathrm{TPa}^{-1}$ and $-10.0 \mathrm{TPa}^{-1} 20$ for $\mathrm{LaNbO}_{4}$, and at $-19.9 \mathrm{TPa}^{-1}$ and $-14.3 \mathrm{TPa}^{-1}$ for $\mathrm{Se}^{21}$ (the alternative, reanalysed, much lower values of reference ${ }^{4}$ for directly measured $\mathrm{LC}$ of $\mathrm{LaNbO}_{4}$ and Se based on ${ }^{20}$ and ${ }^{21}$ are surprising and difficult to reproduce).

Table 1 displays a list of especially interesting examples of materials with NLC and the corresponding values for volume, minimum and maximum LC. A more exhaustive list, additionally including the crystal system and values relative to the volume compressibility is available as Table $\mathrm{S} 2{ }^{19}$. Out of the 38 materials in Table S2, 13 had been identified by Baughman et al. ${ }^{2}$ from the Landolt-Boernstein database ${ }^{22}$. Surprisingly, our methodology identifies 10 additional materials from this same database, most noticeably two with the triclinic crystal system, but also a common material, urea, which exhibits significant NLC at $-36.6 \mathrm{TPa}^{-1}$, or even an element, arsenic ${ }^{1}$, with NLC around $-5 \mathrm{TPa}^{-1}$. The remaining 15 materials have been found via bibliographic searches. Piezoelectric perovskite lead titanate $\left(\mathrm{PbTiO}_{3}\right)$ appears to exhibit a modest NLC, at $-1.0 \mathrm{TPa}^{-1}$. The two unconventional superconductors europium iron arsenide and uranium cobalt germanium also show NLC behaviour, not at standard pressure, but above $3 \mathrm{GPa}$ and $11 \mathrm{GPa}$ respectively. Two phases of the metal oxide framework (MOF) silver hexacyanocobaltate also have significant NLC, up to $-75 \mathrm{TPa}^{-1}$, and many materials with NLC are emerging from the MOF class.

The most important result of this meta-analysis is that common, relatively inexpensive and readily available materials are capable of NLC: we find that extruded polyvinyl chloride

\footnotetext{
${ }^{1}$ NLC in arsenic had been reported as early as $1933^{23} \mathrm{P}$. W. Bridgman, Proceedings of the American Academy of Arts and Sciences 68 (1/13), 27 (1933); R. W. Munn, Journal of Physics Part C Solid State Physics 5 (5), 535 (1972).
} 
$(\mathrm{PVC})^{24}$, extruded poly-propylene (PP $)^{25}$, machine paper $^{26}$, black walnut ${ }^{27}$ and carbon fibre composites $^{28}$ all exhibit NLC.

The mechanism responsible for NLC in these common materials can be understood by considering the wine rack model which is often invoked to explain NLC qualitatively, see for instance ${ }^{6,29}$. Grima et al. ${ }^{30}$ have used a hinged model to analytically derive LC expressions for honeycomb and wine-rack structures. In the next section, we develop an alternative wine-rack model based on beam theory in order to predict optimum geometry. As displayed in Figure. 2, the model consists of an ' $\mathrm{X}$ ' shaped beam -length $L$, area $A$, and second moment of area $I-$ framework in rectangular unit cells where forces/displacements are transmitted at the cell boundary. The resulting equations for compressibilities in the $x$ and $y$ direction are given in equations (4) and consist of a compression term and a bending term,

$$
\begin{aligned}
& \beta_{x}=\frac{2 L \cos \theta \sin \theta}{E A}-\frac{L^{3} \tan \theta\left(\cos ^{2} \theta-\sin ^{2} \theta\right)}{3 E I}, \\
& \beta_{y}=\frac{2 L \cos \theta \sin \theta}{E A}+\frac{L^{3} \cot \theta\left(\cos ^{2} \theta-\sin ^{2} \theta\right)}{3 E I}
\end{aligned}
$$

For $\theta$ at $45^{\circ}$-a square planar structure- no bending is induced in the beams, which results only in a small axial compression, i.e. a small positive linear compressibility. If $\theta$ is less than $45^{\circ}$, the force imbalance results in the beams bending. The beam bending leads to a contraction of the $y$-direction side length of the cell and a subsequent increase in the $x$-direction side length, i.e. the cell has increased in length axially in the $x$-direction under hydrostatic compression as shown in Figure 2(d). Given than axial compression is small compared to the bending displacement for slender beams this effect dominates the deformation of the structure. Figure 3 displays the linear compressibilities for the $\mathrm{X}$ structure in the $x$ and $y$ directions plotted against $\theta$, for a model consisting of cylindrical steel beams of length $100 \mathrm{~mm}$ and radius $5 \mathrm{~mm}$. The structure shows regions of significant negative linear compressibility, with a minimum at $26^{\circ}$, and the largest magnitude NLC is $-23 \%$ of the maximum positive. The results for the $x$ and $y$ linear compressibilities are symmetrical about $45^{\circ}$. The unrealistic divergence for very high unit cell aspect ratios derives from the fact that the beam thickness and consequent overlaps are not taken into account by the theory. Figure $\mathrm{S} 1^{19}$ shows how the maximum NLC value of the structure increases with the slenderness of the beam. Making the $\mathrm{X}$ unit cell model beams more slender increases the magnitude of the predicted NLC because the magnitude of the displacement occurring at the beam free ends increases due to their greater flexibility. Remarkably, the angle $\theta$ 
at which this maximum value occurs remains constant, at $26^{\circ}$ for $\beta_{x}$ and $64^{\circ}$ for $\beta_{y}$, corresponding to unit cell aspect ratios close to 2 . The absolute values of compressibility are low compared to the materials discussed above due to the low relative density of the slender-beam structures.

The mechanism provided by this model explains why materials with significant amount of imperfectly aligned fibres/molecules might have NLC. In this group of materials the imperfectly aligned fibres/molecules behave as the beams in the scissor model, promoting NLC behaviour. A misalignment of $26^{\circ}$ maximises the effect, but Figure 3 shows that some NLC can be achieved with misalignment varying between $0^{\circ}$ and $45^{\circ}$.

For carbon fibre composites this mechanism is present at the macro-scale due to the alignment of the high modulus carbon fibres within the matrix, potentially in both the in-plane and the through-thickness directions depending on the carbon fibre alignment. It leads to large NLC, as can be seen in Figure S3 for a stack of carbon fibre laminae oriented at $37^{\circ}$.

In paper, this mechanism is present at the micro-scale where the paper fibres are given orientation by the rolling process used in their production. Depending on the fibre orientation imparted by the rolling process it may be possible to generate NLC in both the in-plane and through-thickness directions.

In PVC and PP the mechanism occurs at the nano-scale where the extrusion process results in imperfectly aligned polymer chains. In general, extruded polymers show a highly anisotropic linear compressibility, with a significantly reduced magnitude in the direction of extrusion due to the imperfect alignment in the polymer chains causing them to behave in a similar manner to the fibres in the carbon fibre composite and paper.

Considering these results it is clear that NLC is much more common than previously expected and may be found in a wide variety of materials, composites and structures which have a degree of orientation imparted by their production process. The mechanism responsible for NLC in these is very similar to the wine-rack observed in crystalline frameworks ${ }^{7}$. As such the understanding of this unusual property is more important than previously considered as it may already be commonplace in many engineering situations where a complete understanding of constituent material properties is essential.

Due to the constraint of using rare, single crystals, the applications of NLC suggested by previous studies (for instance but not exclusively ${ }^{2,7}$ ) have necessarily been limited in scope. The 
main suggestions have been to use materials with extremal linear compressibilities, either negative or positive, to design pressure sensitive sensors and actuators for use in regions of hydrostatic pressure, such as underwater or in pressure vessels. However, now that NLC has been identified in common materials, the potential for application has increased. Given that the property requires hydrostatic stress to generate an effect, any applications that produce a triaxial stress regime are of interest. Potential areas therefore include crack propagation in composites where the region ahead of a crack tip is placed in triaxial tension. If this effect leads to a natural extension of a composite component in a predetermined direction this may provide extra resistance to crack propagation. Short fibre or nanotube composites might be used to provide this effect with the fibres oriented using flow of the matrix material during cure, which can be achieved using a variety of common moulding processes. Another potential application is in the production of braided tubes and hoses that grip fittings when loaded in tension. The large positive Poisson's ratio of the braid would cause the tube diameter to increase upon compression and decrease under tensile load, allowing for an easy press fit and a secure junction if the tube is pulled. This may have applications in fluid transport pipes and lug jointed carbon fibre tubes such as those found in high performance sailing and cycling equipment. Also of interest is the potential for increased indentation resistance: if a small region of a material is indented then the material directly beneath the indenter is compressed, but if the surrounding material prevents any lateral Poisson's expansion this results in an area of hydrostatic compression directly below the indenter. This effect is similar to that seen in constrained hydrostatic compression test. If the material being indented displays NLC aligned with the axis of indentation then the longitudinal expansion occurring due to the hydrostatic compression could be used to directly resist the indentation, or if the NLC is aligned perpendicular to the axis of indentation then the increased lateral deformation may cause increased indentation resistance.

In conclusion, this paper has identified several relatively common materials that display NLC. These materials are currently in use in real, "engineering" applications, which raises the prospect of application for NLC. We also present a simple 2D analytical model that describes well the underlying mechanism of NLC. Finally, several potential applications for NLC materials were discussed, which hopefully could lead to increased interest in the field from engineers and scientists working with practical applications as well as academics. 
Acknowledgements: Funding was provided by EPSRC Grant EP/G064601/1

\section{Bibliography}

P. W. Bridgman, Proc. Natl. Acad. Sci. U.S.A. 8, 361 (1922).

R. H. Baughman, S. Stafstrom, C. X. Cui, and S. O. Dantas, Science 279 (5356), 1522 (1998).

A. E. Aliev, J. Y. Oh, M. E. Kozlov, A. A. Kuznetsov, S. L. Fang, A. F. Fonseca, R. Ovalle, M. D. Lima, M. H. Haque, Y. N. Gartstein, M. Zhang, A. A. Zakhidov, and R. H. Baughman, Science 323 (5921), 1575 (2009).

A. L. Goodwin, D. A. Keen, and M. G. Tucker, Proc. Natl. Acad. Sci. U.S.A. 105 (48), 18708 (2008).

A. D. Fortes, E. Suard, and K. S. Knight, Science 331 (6018), 742 (2011).

A. B. Cairns, J. Catafesta, C. Levelut, J. Rouquette, A. van der Lee, Lars Peters, A. L.

Thompson, V. Dmitriev, J. Haines, and A. L. Goodwin, Nature Materials 12 (3), 212 (2013).

A. B. Cairns and A. L. Goodwin, PCCP in press (2015).

F.-X. Coudert, PCCP 15 (38), 16012 (2013).

R. Lakes, Science 235 (4792), 1038 (1987).

T. A. Mary, J. S. O. Evans, T. Vogt, and A. W. Sleight, Science 272 (5258), 90 (1996).

R. Aylo, P. P. Banerjee, and G. Nehmetallah, J. Opt. Soc. Am. B 27 (3), 599 (2010).

R. S. Lakes, T. Lee, A. Bersie, and Y. C. Wang, Nature 410 (6828), 565 (2001).

R. H. Baughman, D. S. Galvao, C. Cui, and S. O. Dantas, Chem. Phys. Lett. 269 (3-4), 356 (1997); H. C. Hsueh, C. C. Lee, C. W. Wang, and J. Crain, Physical Review B 61 (6), 3851 (2000).

L. F. Braganza and D. L. Worcester, Biochemistry 25 (23), 7484 (1986); R. H.

Baughman, Nature 425 (6959), 667 (2003); S. H. White and G. I. King, Proc. Natl. Acad. Sci. U.S.A. 82 (19), 6532 (1985). J. N. Grima, D. Attard, and R. Gatt, Physica Status Solidi B 245 (11), 2405 (2008).

A. M. Skvortsov, L. I. Klushin, and F. A. M. Leermakers, J. Chem. Phys. 126 (2) (2007);

E. V. Vakarin, Y. Duda, and J. P. Badiali, J. Chem. Phys. 124 (14) (2006).

J. F. Nye, Physical properties of crystals. (Clarendon press, Oxford, 1957).

A. Marmier, Z. A. D. Lethbridge, R. I. Walton, C. W. Smith, S. C. Parker, and K. E.

Evans, Comput. Phys. Commun. 181 (12), 2102 (2010).

See Supplemental Material at [URL will be inserted by publisher] for a more detailed description of the beam model and an expanded list of the 38 NLC capable materials. J. W. E. Mariathasan, L. W. Finger, and R. M. Hazen, Acta Crystallographica Section B 41 (JUN), 179 (1985).

D. R. McCann, L. Cartz, R. E. Schmunk, and Y. D. Harker, J. Appl. Phys. 43 (4), 1432 (1972).

A. G. Every and A. K. McCurdy, in Landolt-Börnstein, Numerical Data and Functional Relationships in Science and Technology (Springer Verlag, Berlin, 1993), Vol. 29a. P. W. Bridgman, Proceedings of the American Academy of Arts and Sciences 68 (1/13), 27 (1933); R. W. Munn, Journal of Physics Part C Solid State Physics 5 (5), 535 (1972).

F. F. Rawson and J. G. Rider, Journal of Physics D-Applied Physics 7 (1), 41 (1974). 
O. K. Chan, F. C. Chen, C. L. Choy, and I. M. Ward, Journal of Physics D-Applied Physics 11 (5), 617 (1978). R. W. Mann, G. A. Baum, and C. C. Habeger, IPC Technical Paper Series 84 (1979). D. E. Kretschmann, in Wood Handbook, Wood as an Engineering Material, General Technical Report FPL-GTR-190 (Department of Agriculture, Forest Service, Forest Products Laboratory, Madison, WI, 2010). D. Roylance, http://web.mit.edu/course/3/3.11/www/modules/plate.html (1996). J. M. Ogborn, I. E. Collings, S. A. Moggach, A. L. Thompson, and A. L. Goodwin, Chemical Science 3 (10), 3011 (2012). J. N. Grima, D. Attard, R. Caruana-Gauci, and R. Gatt, Scripta Mater. 65 (7), 565 (2011). 


\section{Figure captions}

Figure 1. NLC and conventional materials under compressive pressure.

Figure 2. (a) X structure with unit cell shown in red (dashed), b) under uniform hydrostatic compression $\mathrm{P}$, c) with hydrostatic pressure resolved into components acting on the cell boundary, (d) Beam in bending shown overlaid on original structure showing extension in $\mathrm{x}$ direction due to bending imparted by resultant normal force $\mathrm{F}_{\mathrm{n}}$.

Figure 3. Linear compressibilities for 2D X unit cell model with slender beams ( $E=200 \mathrm{GPa}$, $l=100 \mathrm{~mm}, r=5 \mathrm{~mm})$ : full line $\beta_{y}$, dashed line $\beta_{x}$. 
TABLE 1. Compressibilities $\left(\mathrm{TPa}^{-1}\right)$ of selected common materials.

\begin{tabular}{|c|c|c|c|}
\hline Name & $\beta_{\mathrm{v}}$ & $\beta_{\min }$ & $\beta_{\max }$ \\
\hline Drawn PP ${ }^{25}$ & 590.9 & -42.37 & 316.63 \\
\hline Drawn PVC ${ }^{24}$ & 195.50 & -15.15 & 105.30 \\
\hline Machine paper ${ }^{26}$ & 1490 & -175 & 1520 \\
\hline Black walnut ${ }^{27}$ & 978.70 & -9.96 & 819.10 \\
\hline Carbon fibre $^{28}$ & 105.62 & -69.43 & 113.17 \\
\hline
\end{tabular}

